\title{
Caries y salud bucal, percepciones acerca de la enfermedad
}

\author{
Natalia Odeth Santos Madrigal, ${ }^{1}$ \\ Alejandra Moreno-Altamirano, ${ }^{2}$ \\ Norma Laura Lara Flores,
}

\section{Resumen}

La caries dental es una enfermedad con elevada prevalencia a nivel mundial, su identificación y tratamiento es fundamental en edades tempranas. Objetivo: Medir la prevalencia de caries dental en niñas y niños en edad preescolar y comprender su relación con la percepción de sus madres, respecto a su salud bucal. Materiales y métodos. La metodología del estudio fue mixta. Se realizó un estudio transversal en 213 preescolares, de 3 a 6 años de edad que fueron revisados clínicamente para determinar la prevalencia de caries y el índice ceo-d, de acuerdo con estándares de la OMS. Se utilizó un cuestionario y una entrevista cualitativa para conocer la percepción de la salud bucal de las madres y sus hijos. Resultados. La prevalencia de caries dental observada fue de $69 \%$ con un índice ceo-d de 4,34. Más de la mitad de las madres, $53,4 \%$ calificaron la salud bucal de sus hijos como "regular". Las madres identificaron la presencia de caries dental en sus hijas e hijos, pero no en etapas iniciales. La percepción de las madres acerca de la salud bucal de sus hijos estuvo asociada con baja escolaridad y limitado acceso a los servicios odontológicos derivados de sus condiciones socioeconómicas. Conclusiones. La prevalencia de caries dental en niños en edad preescolar se asoció con sus condiciones de vida y la precepción de la salud bucal de sus madres. Son necesarias estrategias de promoción y prevención, orientadas profesionalmente, que enfaticen la importancia de la salud bucal en el desarrollo de los preescolares.

Palabras clave: caries, prevalencia, percepción, salud bucal.

\footnotetext{
${ }^{1}$ Maestria en Medicina Social. División de Ciencias Biológicas y de la Salud Universidad Autónoma Metropolitana. Calada del Hueso 1100, C.P.14387 Ciudad de México. ${ }^{2}$ Departamento de Salud Pública y del Programa de Maestría y Doctorado en Ciencias Médicas, Odontológicas y de la Salud. Facultad de Medicna Universidad Nacional Autónoma de México. Av. Universidad 3000, Ciudad de México, C.P. 04510.

${ }^{3}$ Departamento de Atención a la Salud. División de Ciencias Biológicas y de la Salud Universidad Autónoma Metropolitana. Calada del Hueso 1100, C.P.14387 Ciudad de México.
} 
Artigo original

\section{Cárie e saúde bucal, percepções sobre a doença}

\section{Resumo}

A cárie dentária é uma doença com alta prevalência em todo o mundo, sua identificação e tratamento são essenciais desde tenra idade. Objetivo: Medir a prevalência de cárie dentária em meninas e meninos em idade pré-escolar e compreender sua relação com a percepção de suas mães em relação à sua saúde bucal. Materiais e métodos. A metodologia do estudo foi mista. Foi realizado um estudo transversal em 213 pré-escolares, de 3 a 6 anos de idade, revisados clinicamente para determinar a prevalência de cárie e o índice ceo-d, de acordo com os padrões da OMS. Um questionário e uma entrevista qualitativa foram utilizados para descobrir a percepção da saúde bucal das mães e de seus filhos. Resultados. A prevalência de cárie dentária observada foi de $69 \%$, com um índice ceo-d de 4,34. Mais da metade das mães, 53,4\% classificou a saúde bucal de seus filhos como "regular". As mães identificaram a presença de cárie dentária em suas filhas e filhos, mas não nos estágios iniciais. A percepção das mães sobre a saúde bucal de seus filhos esteve associada à baixa escolaridade e acesso limitado a serviços odontológicos derivados de suas condições socioeconômicas. Conclusões. A prevalência de cárie dentária em crianças em idade pré-escolar associou se com às condições de vida e à percepção de saúde bucal das mães. São necessárias estratégias de promoção e prevenção com orientação profissional que enfatizem a importância da saúde bucal no desenvolvimento dos pré-escolares.

Palavras-chave: cárie, prevalência, percepção, saúde bucal.

Original article

\section{Caries and oral health, perceptions about the disease}

\begin{abstract}
Dental caries is a major public health problem globally and is the most widespread non-communicable disease, it's identification and treatment is essential at an early age. Objective: To measure the prevalence of dental caries in preschool girls and boys and to understand its relationship with the mother's perception of their oral health. Materials and methods: this was a
\end{abstract}

mixed methods study. To determine the prevalence of dental caries def-s index was used. Oral review of 213 preschoolers following the WHO standards was performed. A questionnaire was filled out by the mothers and they were also interviewed to know their perceptions of the oral health of their children. Results. The prevalence of dental caries observed was $69 \%$ with a def-s index of 4.34 . More than half of the mothers, $53.4 \%$ rated the oral health of their children 
as "regular". The mothers identified the presence of dental caries in their daughters and sons, but not in the initial stages. Mothers' perception of their children's oral health was associated with low schooling and limited access to dental services derived from their socioeconomic conditions. Conclusions. The prevalence of dental caries in

\section{Introducción}

La Salud y la enfermedad son procesos con manifestaciones orgánicas que ocurren en individuos pertenecientes a grupos sociales definidos, son el resultado de la interacción entre factores biológicos y un contexto social, dependen de las percepciones e interpretaciones que los individuos y grupos dan a estos. Ambos elementos están relacionados con la concepción del mundo que un grupo o un individuo tienen de acuerdo con su inserción social y sus prácticas cotidianas. ${ }^{1}$ El concepto de salud-enfermedad-atención (PSEA), reconoce que se trata de procesos que se modifican en cada sociedad, que obedece a múltiples relaciones entre el individuo y su entorno, y que la percepción de la enfermedad depende de la cultura de cada grupo social., 3

México es un país donde las diferencias sociales se ven reflejadas en el perfil epidemiológico de la población, distinguido por las enfermedades de la pobreza donde es común la superposición de enfermedades infectocontagiosas a las cuales se suman las crónico-degenerativas. ${ }^{4,5}$ Los problemas bucodentales se encuentran dentro de dichas enfermedades, su incidencia se preschool children was associated with their living conditions and the mother's perception of oral health. Professionally oriented prevention and prevention strategies are needed that emphasize the importance of developing oral health in preschoolers.

Keywords: dental caries, prevalence, perception, oral health.

ve agravada por condiciones de vida precarias y por la falta de acceso a una atención oportuna. ${ }^{6}$

La caries dental es una enfermedad infecciosa de origen multifactorial que puede iniciar en edades tempranas con la erupción dentaria, se reconoce que afecta la salud general y que altera la calidad de vida. La generación de caries durante la infancia, se ha asociado con hábitos en la lactancia materna, el uso de biberón nocturno, el uso del chupón, una dieta rica en carbohidratos, con aspectos frecuentemente determinados por las condiciones de vida de los niños y sus familias, tales como bajo nivel educativo y ausencia de hábitos higiénicos en general. $^{7}$

Estudios realizados en preescolares mexicanos de 3 a 5 años de edad, han reportado una prevalencia de caries en la dentición primaria del 47,6\% en niños y niñas del estado de Campeche y considerando como pérdida dental la afectación de la superficie de la corona mayor a $2 / 3$ partes, $11 \%$ de los niños en este estudio tuvo algún diente perdido. ${ }^{8}$ En el Estado México (entidad federativa periférica a la capital), la prevalencia de caries reportada fue del 69,5\% y 
considerando como indicador el ceo-d se encontró un promedio de 3,44 dientes afectados en niños y 3,62 en niñas de cuatro y cinco años de edad. ${ }^{9}$ Otro estudio realizado en Milpa Alta, alcaldía rural de la Ciudad de México, mostró que el promedio de dientes afectados, ceo-d fue de 3,9 en general en todos los niños revisados, siendo éste de 2,2 a los tres años, 4,2 a los cuatro años y 4,7 a los cinco años. ${ }^{10}$ Los estudios anteriores, indican que la caries dental es una enfermedad de inicio temprano, en la cual las lesiones cariosas aumentan y se agravan conforme a la edad, con la consecuente pérdida de uno o más dientes, generando un estado de dolor y malestar, limitando la alimentación, alterando la oclusión, repercutiendo en la salud de la dentición permanente.

En este contexto, el papel de las madres es considerado muy influyente en el cuidado de la salud bucal y la prevención de las enfermedades de los niños ya que son ellas quienes, en primera instancia, las identifican y tratarán de solucionarlas. Culturalmente la madre es considerada clave en la reproducción de la sociedad y de la cultura. ${ }^{11}$ Las madres que pertenecen a grupos en condiciones de pobreza se exponen junto a sus hijos a factores relacionados con la aparición de la caries dental. Su percepción respecto a la caries y la salud bucodental es relevante, debido a que son ellas quienes por lo general, están a cargo del cuidado de los hijos, la observación de su crecimiento y desarrollo, de su alimentación, de la formación de hábitos y de su salud. ${ }^{12}$

El objetivo del presente trabajo fue medir la prevalencia de caries dental en niñas y niños en edad preescolar, y comprender su relación con la percepción de sus madres, respecto a su salud bucal.

\section{Materiales y métodos}

El trabajo contó con el aval del Comité Académico de la Maestría en Medicina Social de la Universidad Autónoma Metropolitana, el cual verificó el respeto y observancia de todas las consideraciones científicas y éticas pertinentes, así como de los principios éticos de la declaración de Helsinki, pues la investigación no expuso a riesgos ni daños a los sujetos que participaron en ella; resguardando el cumplimiento de los principios éticos de autonomía, no maleficencia, beneficencia y justicia. En todos los casos se entregaron consentimientos informados a las madres de los preescolares para su revisión y a ellas para su participación. El material utilizado en el estudio, así como los gastos operativos, fueron costeados por las autoras.

Se realizó un estudio transversal con un diseño mixto; cuantitativo y cualitativo. Se trabajó con el total de la población de preescolares $(n=213)$ entre 3 y 6 años de edad del Jardín de niños María Patiño Viuda de Olmedo, de la Secretaria de Educación Pública, ubicado en la alcaldía de Xochimilco en la Ciudad de México, en el año 2011. A toda la población preescolar, se le realizó una revisión bucal siguiendo los criterios recomendados por la OMS para el levantamiento de encuestas de caries dental. ${ }^{13}$ Se calculó el índice ceo-d considerando que la OMS clasifica la escala de gravedad de la afección en cinco niveles: muy bajo (00-1,1), bajo $(1,2-2,6)$, moderado $(2,7-4,4)$, alto $(4,5-6,5) \mathrm{y}$, muy alto $(>6,5)$; la metodología señala la experiencia de caries presente y pasada, pues considera las lesiones, los tratamientos observados y se calculó la prevalencia de caries dental.

A las madres de los niños les fue aplicado 
un cuestionario de 37 preguntas cerradas divididas en cinco bloques, para conocer la percepción de su salud bucal y la de sus hijos, sus respuestas se agruparon en tres categorías: buena, regular y mala. Así mismo, se les preguntó sobre características sociodemográficas, el uso de servicios de salud y odontológicos, las prácticas de cuidado bucal de sus hijos y sobre los patrones de consumo de alimentos cariogénicos.

El análisis estadístico se realizó empleando el paquete SPSS ${ }^{\circledR}$ versión 20 . Se obtuvieron frecuencias simples, relativas y promedios. Los datos permitieron definir el perfil de madres en cuanto a sus características económicas, sociales y acceso a servicios de salud, además de la percepción sobre la su propia salud bucal y de sus hijos. Para asociar la prevalencia de caries y el índice ceo-d con el perfil de la madre, la percepción sobre la salud bucodental de sus hijos y características de los niños se usaron las pruebas de Chi cuadrado, $t$ de Student y análisis de varianza como pruebas de hipótesis.

Se realizó una entrevista semiestructurada a una submuestra de 12 madres, cuatro por cada categoría de percepción de la salud bucal de sus hijos (buena, regular y mala). La entrevista se orientó por las siguientes dimensiones: a) Percepciones, significaciones y valor social atribuidos a la boca, b) Significación que la salud y enfermedad bucal representan para las madres y sus familias, c) Manejo del dolor dental por parte de la madre como evento que activa la valoración de la propia salud y la de su familia, especialmente de sus hijos, d) Estrategias de educación y de cuidado de las enfermedades de la boca,

e) Empleo de dulces, golosinas y otros alimentos cariogénicos como recompensas, f) Experiencias de las madres frente a la atención y los servicios odontológicos, g) Fuentes de sus conocimientos.

Las entrevistas se transcribieron y se empleó el análisis de contenido para identificar y clasificar las unidades de análisis, palabras, frases, párrafos de la entrevista según las dimensiones de las respuestas culturales (percepción). Se aplicó el análisis etnográfico, que consiste en describir e interpretar, en su contexto, al grupo, sistema social o cultural con el cual se trabaja, en este caso, las madres de los preescolares. ${ }^{14,15}$ De este proceso de análisis surgieron las siguientes categorías: a) Respuestas culturales sobre el proceso salud enfermedad bucodental (PSEBD), b) Percepción de la boca, c) Conocimientos y experiencias acerca las de las enfermedades bucodentales (EBD), d) Estrategias de educación y atención de las enfermedades bucodentales (EBD), e) Manejo del dolor dental, que activa la valoración de la Salud Bucodental, y f) Importancia de los servicios odontológicos.

\section{Resultados}

\section{Preescolares}

La edad, sexo, última visita al dentista, motivo de la consulta y tipo de servicio de salud utilizado por los preescolares se muestran en la Tabla 1. En cuanto a edad la moda fue de cinco años, el $29 \%$ nunca había visitado al Odontólogo. El 44\% de las madres respondió que sus hijos realizan cepillado dental dos veces al día.

La prevalencia de caries dental fue de $69 \%$ en toda la población; el 70,5\% de las niñas 
Tabla 1: Prevalencia e indice de dientes primarios cariados, indicado para extracción y obturados (ceo) de los preescolares del Jardín de niño Ma. Patiño Vda. De Olmedo según las características demográficas y odontológicas (2011).

\begin{tabular}{|c|c|c|c|c|c|c|}
\hline Variables & $\mathbf{N}$ & $\begin{array}{c}\text { Prevalencia } \\
\text { de caries } \\
\text { n (\%) }\end{array}$ & $\begin{array}{c}\mathrm{c} \\
\mathrm{X}(\mathrm{DE})\end{array}$ & $\begin{array}{c}e \\
x(D E)\end{array}$ & $\begin{array}{c}\mathbf{0} \\
X(\mathrm{DE})\end{array}$ & $\begin{array}{c}\text { ceo } \\
X(\mathrm{DE})\end{array}$ \\
\hline Total & 213 & $147(69)$ & $3,63(3,73)$ & $0,02(2,27)$ & $0,66(1,54)$ & $4,34(4,12)$ \\
\hline \multicolumn{7}{|c|}{ Sexo } \\
\hline Femenino & $101(47,42)$ & $79(70,5)$ & $3,70(3,57)$ & $0,04(0,38)$ & $0,55(1,55)$ & $4,30(4,05)$ \\
\hline Masculino & $112(52,58)$ & $68(67,3)$ & $3,55(3,90)$ & $0,00(0,00)$ & $0,78(1,54)$ & $4,39(4.21)$ \\
\hline$F(p)$ & & & $0,08(0,78)$ & $0,90(0,34)$ & $1,16(0,28)$ & $0,03(0,85)$ \\
\hline \multicolumn{7}{|c|}{ Edad del preescolar } \\
\hline 4 años & $26(12,21)$ & $17(54,8)$ & $2,39(3,40)$ & $0,13(0,71)$ & $0,10(0,30)$ & $2,64(3,96)$ \\
\hline 5 años & $119(55,87)$ & $80(69,6)$ & $3,86(3,82)$ & $0,00(0,00)$ & $0,66(1,52)$ & $4,54(4,23)$ \\
\hline 6 años & $68(31,92)$ & $50(74,6)$ & $3,81(3,61)$ & $0,00(0,00)$ & $0,93(1,85)$ & $4,70(3,8)$ \\
\hline$F(p)$ & & & $2.02(0,13)$ & $2,9 \quad(0,05)$ & $3,09(0,04)$ & $3,20(0,04)$ \\
\hline \multicolumn{7}{|c|}{ Última visita del niño al dentista } \\
\hline Menos de 3 meses & $42(19,72)$ & $10(23,81)$ & $4,70(3,77)$ & $0,00(0,00)$ & $1,50(2,34)$ & $6,26(4,24)$ \\
\hline De 3 a 6 Meses & $41(19,25)$ & $11(2,83)$ & $3,44(3,42)$ & $0,10(0,62)$ & $1,15(1,89)$ & $4,70(3,90)$ \\
\hline De 7 a 12 meses & $26(12,21)$ & $7(26,92)$ & $3,88(3,98)$ & $0,00(0,00)$ & $0,58(1,39)$ & $4,57(4,09)$ \\
\hline Más de un año & $42(19,72)$ & $13(30,95)$ & $3,62(3,90)$ & $0,00(0,00)$ & $0,24(0,65)$ & $3,38(4,22)$ \\
\hline Nunca ha ido al dentista & $62(29,11)$ & $25(40,32)$ & $2,92(3,62)$ & $0,00(0,00)$ & $0,10(0,34)$ & $3,01(3,68)$ \\
\hline$F(p)$ & & & $1,59(0,19)$ & $1,05(0,38)$ & $7,85(0,01)$ & $4,37(0,02)$ \\
\hline \multicolumn{7}{|c|}{ Motivo de la consulta* } \\
\hline Alguna molestia al dolor & $44(20,66)$ & $7(15,91)$ & $5,45(4,10)$ & $0,00(0,00)$ & $0,84(1,49)$ & $6,36(4,07)$ \\
\hline $\begin{array}{l}\text { Continuar con algún } \\
\text { tratamiento }\end{array}$ & $22(10,33)$ & $5(22,73)$ & $3,77(3,14)$ & $0,00(0,00)$ & $2,32(2,85)$ & $6,27(4,35)$ \\
\hline Prevención & $67(31,46)$ & $20(29,85)$ & $3,24(3,48)$ & $0,00(0,00)$ & $0,58(1,43)$ & $3,82(3,80)$ \\
\hline Estética & $6(2,82)$ & $2(33,33)$ & $2,17(3,06)$ & $0,00(0,00)$ & $0,67(1,21)$ & $2,83(3,12)$ \\
\hline Otra no especificada & $12(5,63)$ & $7(58,33)$ & $3,08(4,20)$ & $0,33(0,15)$ & $0,17(0,57)$ & $3,58(4,81)$ \\
\hline Nunca ha ido al dentista & $62(29,11)$ & $25(40,32)$ & $2,95(3,59)$ & $0,00(0,00)$ & $0,13(0,42)$ & $3,08(3,69)$ \\
\hline$F(p)$ & & & $3,03(0,01)$ & $3,55(0,04)$ & $8,06(0,01)$ & $5,15(0,01)$ \\
\hline
\end{tabular}


Tabla 1: Prevalencia e indice de dientes primarios cariados, indicado para extracción y obturados (ceo) de los preescolares del Jardín de niño Ma. Patiño Vda. De Olmedo según las características demográficas y odontológicas (2011). Cont.

\begin{tabular}{|c|c|c|c|c|c|c|}
\hline Variables & $\mathbf{N}$ & $\begin{array}{c}\text { Prevalencia } \\
\text { de caries } \\
\text { n }(\%)\end{array}$ & $\begin{array}{c}\mathrm{c} \\
\mathrm{X}(\mathrm{DE})\end{array}$ & $\begin{array}{c}\mathrm{e} \\
X(\mathrm{DE})\end{array}$ & $\begin{array}{c}0 \\
X(D E)\end{array}$ & $\begin{array}{c}\text { ceo } \\
X(D E)\end{array}$ \\
\hline Total & 213 & $147(69)$ & $3,63(3,73)$ & $0,02(2,27)$ & $0,66(1,54)$ & $4,34(4,12)$ \\
\hline \multicolumn{7}{|c|}{ Tipo se servicio odontológico utilizado } \\
\hline $\begin{array}{l}\text { Instituto Mexicano del } \\
\text { Seguro Social (IMSS) }\end{array}$ & $12(5,63)$ & $4(33,33)$ & $3,00(3,95)$ & $0,00(0,00)$ & $0,25(0,62)$ & $3,25(4,22)$ \\
\hline $\begin{array}{l}\text { Instituto de Seguridad y } \\
\text { Servicios Sociales de los } \\
\text { Trabajadores del Estado } \\
\text { (ISSSTE) }\end{array}$ & $6(2,82)$ & $3(50,00)$ & $2,17(2,85)$ & $0,67(1,63)$ & $0,17(0,40)$ & $3,00(4,19)$ \\
\hline $\begin{array}{l}\text { Secretaria de la Defensa } \\
\text { Nacional (SEDENA) }\end{array}$ & $1(0.47)$ & $0(0,00)$ & $10,00(0,00)$ & $0,00(0,00)$ & $0,00(0,00)$ & $10,00(0,00)$ \\
\hline $\begin{array}{l}\text { Petróleos Mexicanos } \\
\text { (PEMEX) }\end{array}$ & $1(0,47)$ & $1(100,00)$ & $0,00(0,00)$ & $0.00(0,00)$ & $0,00(0,00)$ & $0,00(0,00)$ \\
\hline Privado & $78(36,62)$ & $17(21,79)$ & $3,92(3,55)$ & $0,00(0,00)$ & $1,01(1,97)$ & $4,94(4,02)$ \\
\hline Secretaría de Salud & $27(12,68)$ & $10(37,04)$ & $4,00(4,30)$ & $0,00(0,00)$ & $0.81(1,44)$ & $4,85(4,76)$ \\
\hline Otro no especificado & $25(11,74)$ & $6(24,00)$ & $4,64(3,90)$ & $0,00(0,00)$ & $1,12(2,00)$ & $5,96(3,86)$ \\
\hline Nunca ha ido al dentista & $61(28,64)$ & $25(40,98)$ & $2,89(3,58)$ & $0,00(0,00)$ & $0,13(0,42)$ & $3,01(3,69)$ \\
\hline Sin servicio & & & $4,00(4,24)$ & $0,00(0,00)$ & $0,00(0,00)$ & $4,00(4,24)$ \\
\hline$F(p)$ & & & $1,27(0,25)$ & $4,95(0,04)$ & $2,05(0,04)$ & $2,18(0,03)$ \\
\hline \multicolumn{7}{|c|}{ Veces de cepillado al día } \\
\hline 0 & $18(08,5)$ & $3(16,67)$ & $6,56(4,32)$ & $0,00(0,00)$ & $0,44(0,78)$ & $7,16(4,56)$ \\
\hline 1 & $62(29,1)$ & $21(33,87)$ & $3,39(3,63)$ & $0,00(0,00)$ & $0,34(1,11)$ & $3,77(3,73)$ \\
\hline 2 & $94(44,1)$ & $30(31,91)$ & $3,76(3,76)$ & $0,04(0,41)$ & $0,30(1,54)$ & $4.60(4,17)$ \\
\hline 3 & $39(18,3)$ & $12(30,77)$ & $2,36(2,81)$ & $0,00(0,00)$ & $0,45(2,22)$ & $3,30(3,86)$ \\
\hline$F(p)$ & & & $5.64(0,01)$ & $0,41(0,14)$ & $1,72(0,16)$ & $4,35(0,05)$ \\
\hline \multicolumn{7}{|c|}{ Consumo de dulces } \\
\hline Todos los días & $38(17,8)$ & $8(21,05)$ & $4,84(3,59)$ & $0,11(0,64)$ & $0,17(1,45)$ & $5,73(4,13)$ \\
\hline La mayoría de los días & $89(41,8)$ & $27(30,34)$ & $3,94(3,89)$ & $0,00(0,00)$ & $0,73(1,79)$ & $4,68(4,43)$ \\
\hline Una vez por semana & $53(24,9)$ & $18(33,96)$ & $2,55(3,14)$ & $0,00(0,00)$ & $0,43(1,04)$ & $3,01(3,27)$ \\
\hline Rara vez & $43(20,2)$ & $12(27,91)$ & $3,21(4,00)$ & $0,00(0,00)$ & $0,82(1.64)$ & $4,06(4,01)$ \\
\hline Nunca & $1(0,5)$ & $0(0,00)$ & $1,00(0,000)$ & $0,00(0,00)$ & $0,00(0,00)$ & $1,00(0,00)$ \\
\hline$F(p)$ & & & $2,56(0,03)$ & $1,45(0,33)$ & $0,46(0,76)$ & $2,90(0,02)$ \\
\hline
\end{tabular}


y el $69 \%$ de los niños presentaron caries dental; no se encontraron diferencias estadísticamente significativas en cuanto al sexo. La relación de la prevalencia de la caries dental con las variables evaluadas de los niños se muestra en la Tabla 1 y 1 bis. La prevalencia más alta se encuentra en los niños de 4 años (42,3\%) y en los niños que nunca han ido al Odontólogo $(40,32 \%)$.

El índice ceo-d fue de 4,34 (Tabla 1). La relación entre la edad y el índice ceo-d fue significativa $(p<0,05)$ en los niños de 4 años fue de 2,64, en los de 5 años 4,55 y los de 6 años. ${ }^{4,7}$

\section{Madres de preescolares}

El 47 y $46 \%$ de las madres consideraron la salud bucal de sus hijos como buena y regular respectivamente, la mayoría estudió únicamente hasta el nivel de secundaria y el $44,6 \%$ carece de seguridad social (Tabla 2 y 2 bis).

La percepción de las madres sobre la presencia de la enfermedad caries en sus hijos no se encontró asociada con la prevalencia, pero si con el índice ceo-d de éstos. Las madres que percibieron como buena la salud bucal de sus hijos corresponden a los preescolares que presentaron un índice ceo-d de 1,83, las que la perciben como regular, 5,57 y como mala 5,56, esta asociación fue estadísticamente significativa $\quad(p<0,05)$ (Tabla 2).

Se observó una diferencia que, sin ser estadísticamente significativa, muestra que la escolaridad de la madre se relaciona con la prevalencia de caries dental pues a menor escolaridad se presenta mayor prevalencia de caries dental en sus hijos (Tabla 2bis). El grado de escolaridad de los padres es mayor que el de las madres, pues la mayoría de ellos alcanzan el nivel bachillerato. La ocupación de los padres se asoció con la prevalencia de caries $(\mathrm{p}<0,05)$ lo cual, indica que la ocupación del padre tiene un efecto negativo sobre la salud bucodental de sus hijos pues aquellos que tienen un empleo no calificado, comerciantes y empleados libres, tienen hijos con mayor prevalencia de caries dental que los padres que tienen un empleo estable (tienen un contrato por tiempo indeterminado y prestaciones (Tabla 2bis).

En cuanto a las condiciones de vida, se observó que cuando es mayor la escolaridad del padre, es menor el índice ceo-d. Padres que cuentan únicamente con la educación primaria incompleta tienen hijos con 11 dientes cariados en promedio mientras que los hijos de padres con licenciatura tenían tres. El tipo de ocupación mostró relación con el índice ceo $(\mathrm{p}<0,05)$ (Tablas 2bis y 3$)$.

Respecto a los cuidados dentales, se observó que a menor número de cepillados al día, aumenta el número de dientes cariados (Tabla 3).

Para el análisis cualitativo se entrevistaron a cuatro madres para cada grupo analizado: 1) quienes consideraban la salud de su hijos como buena, 2) quienes la consideraron regular y 3) y aquellas que la consideraron mala. Se consideraron sus tiempos y disponibilidad para participar en esta parte del estudio (Tabla 4).

Se encontró que, para los tres grupos, la 
Tabla 2: Prevalencia e índice de dientes primarios cariados, indicado para extracción y obturados (ceo) de los preescolares del Jardín de niño Ma. Patiño Vda. De Olmedo según la Percepción de la madre de la SB de su hijo, su estado civil y sus condiciones de vida (2011).

\begin{tabular}{|c|c|c|c|c|c|c|}
\hline Variables & $\mathbf{N}$ & $\begin{array}{l}\text { Prevalencia } \\
\text { de caries } \\
\text { n (\%) }\end{array}$ & $\begin{array}{c}\mathrm{c} \\
\mathrm{X}(\mathrm{DE})\end{array}$ & $\begin{array}{c}\mathrm{e} \\
\mathrm{X}(\mathrm{DE})\end{array}$ & $\begin{array}{c}\mathbf{0} \\
\mathrm{X}(\mathrm{DE})\end{array}$ & $\begin{array}{c}\text { ceo } \\
X(\mathrm{DE})\end{array}$ \\
\hline Total & 213 & $147(69)$ & $3,63(3,73)$ & $0,02(2,27)$ & $0,66(1,54)$ & $4,34(4,12)$ \\
\hline \multicolumn{7}{|c|}{ Percepción de la madre de la SB de su hijo } \\
\hline \multirow[t]{2}{*}{ Muy buena y buena } & $100(47)$ & $52(52,00)$ & $1,05(7,63)$ & $0,00(0,00)$ & $0,68(1,82)$ & $83(3,05)$ \\
\hline & & & $2,27(3,10)$ & $0,00(0,00)$ & $0,47(1,25)$ & $2,82(3,32)$ \\
\hline Regular & $98(46)$ & $81(82,70)$ & $4,73(3,69)$ & $0,04(0,04)$ & $0,75(1,66)$ & $5,57(4,22)$ \\
\hline \multirow[t]{2}{*}{ Mala y muy mala } & $15(7)$ & $14(93,33)$ & $6,58(3,39)$ & $0,00(0,00)$ & $1,25(1,91)$ & $8,08(3,55)$ \\
\hline & & & $5,67(5,50)$ & $0,00(0,00)$ & $0,00(0,00)$ & $5,66(5,50)$ \\
\hline$F(p)$ & & & $10,04(0,00)$ & $0,28(0,38)$ & $2,23(0,06)$ & $10,43(0,00)$ \\
\hline \multicolumn{7}{|c|}{ Estado Civil } \\
\hline Soltera & $31(14,55)$ & $12(5,63)$ & $3,29(4.00)$ & $0,00(0,00)$ & $0,77(1,62)$ & $4,06(4,48)$ \\
\hline Casada & $92(43,19)$ & $32(15,02)$ & $3,41(3,65)$ & $0,04(0,41)$ & $0,70(1,80)$ & $4,20(4,34)$ \\
\hline Divorciada & $3(1,41)$ & $2(0,94)$ & $2,33(4,04)$ & $0,00(0,00)$ & $0,33(0,57)$ & $2,66(4,61)$ \\
\hline Separada & $14(6,57)$ & $4(1,88)$ & $2,93(3,56)$ & $0,00(0,00)$ & $1,29(1,38)$ & $4,35(3,95)$ \\
\hline Unión Libre & $73(34,27)$ & $16(7,51)$ & $4,23(3,76)$ & $0,00(0,00)$ & $0,47(1,15)$ & $4,69(3,75)$ \\
\hline$F(p)$ & & & $0,20(0,93)$ & $1,32(0.26)$ & $1,30(0,27)$ & $1,30(0,27)$ \\
\hline \multicolumn{7}{|c|}{ Escolaridad de la madre } \\
\hline Primaria incompleta & $12(5,6)$ & $11(91,7)$ & $3,58(3,82)$ & $0,00(0,00)$ & $0,75(1,05)$ & $4,33(3,89)$ \\
\hline Primaria completa & $14(6,6)$ & $11(78,6)$ & $6,07(4,71)$ & $0,00(0,00)$ & $0,50(1,09)$ & $6,57(4,61)$ \\
\hline Secundaria & $72(33,8)$ & $53(73,6)$ & $4,21(3,85)$ & $0,00(0,00)$ & $0,76(1,51)$ & $5,02(4,12)$ \\
\hline Bachillerato & $40(18,8)$ & $28(70,0)$ & $3,03(3,22)$ & $0,00(0,00)$ & $0,53(1,78)$ & $3,55(3,94)$ \\
\hline Carrera técnica & $52(24,4)$ & $29(55,8)$ & $2,73(3,32)$ & $0,08(0,55)$ & $0,21(0,80)$ & $3,07(3,73)$ \\
\hline Licenciatura & $23(10,8)$ & $15(65,2)$ & $3,43(3,87)$ & $0,00(0,00)$ & $1,65(2,38)$ & $5,08(4,32)$ \\
\hline $\mathrm{F}(\mathrm{p})$ & & & $2.44(0,03)$ & $0,61(0,69)$ & $3,07(0,01)$ & $2.75(0.02)$ \\
\hline \multicolumn{7}{|c|}{ Escolaridad del padre } \\
\hline Primaria incompleta & $1(0,5)$ & $1(100)$ & $11,00(0,00)$ & $0,00(0,00)$ & $0,00(0,00)$ & $11,00(0,00)$ \\
\hline Primaria completa & $13(6,1)$ & $12(92,3)$ & $6,00(0,00)$ & $0,00(0,00)$ & $0,85(1,14)$ & $6,84(4,20)$ \\
\hline Secundaria & $53(24,9)$ & $38(71,7)$ & $4,38(3.97)$ & $0,00(0,00)$ & $0,30(0,74)$ & $4,71(4,04)$ \\
\hline Bachillerato & $76(35,7)$ & $56(73,7)$ & $3,63(3,49)$ & $0,00(0,00)$ & $0,83(1,94)$ & $4,46(4,12)$ \\
\hline Carrera técnica & $14(6,6)$ & $9(64,3)$ & $2,64(2,89)$ & $0,29(1,06)$ & $1,07(2,05)$ & $4,21(4,87)$ \\
\hline Licenciatura & $20(9,4)$ & $9(45,0)$ & $2,30(3,64)$ & $0,00(000)$ & $0,05(0,22)$ & $2,35(3,67)$ \\
\hline No aplica (no tiene padre) & $36(16,9)$ & $22(61,1)$ & $2,58(3,26)$ & $0,00(0,00)$ & $0,97(1,68)$ & $3,61(3,65)$ \\
\hline$F(p)$ & & & $3,09(0,00)$ & $2,46(0,02)$ & $1,64(0,13)$ & $2,35(0,03)$ \\
\hline
\end{tabular}


Tabla 2: Prevalencia e índice de dientes primarios cariados, indicado para extracción y obturados (ceo) de los preescolares del Jardín de niño Ma. Patiño Vda. De Olmedo según la Percepción de la madre de la SB de su hijo, su estado civil y sus condiciones de vida (2011). Cont.

\begin{tabular}{|c|c|c|c|c|c|c|}
\hline Variables & $\mathbf{N}$ & $\begin{array}{c}\text { Prevalencia } \\
\text { de caries } \\
\text { n }(\%)\end{array}$ & $\begin{array}{c}c \\
X(D E)\end{array}$ & $\begin{array}{c}\mathrm{e} \\
X(\mathrm{DE})\end{array}$ & $\begin{array}{c}\mathbf{0} \\
X(\mathrm{DE})\end{array}$ & $\begin{array}{c}\text { ceo } \\
X(\mathrm{DE})\end{array}$ \\
\hline Total & 213 & $147(69)$ & $3,63(3,73)$ & $0,02(2,27)$ & $0,66(1,54)$ & $4,34(4,12)$ \\
\hline \multicolumn{7}{|c|}{ Ocupación de la madre } \\
\hline Empleada calificada & $34(16)$ & $22(64,7)$ & $3,29(3,65)$ & $0,00(0,00)$ & $0,79(2,01)$ & $4,08(4,35)$ \\
\hline Empleada no calificada & $51(23,9)$ & $32(62,7)$ & $3,06(3,59)$ & $0,08(0,56)$ & $0,57(1,25)$ & $3,72(3,92)$ \\
\hline Comerciante & $27(12,7)$ & $19(70,4)$ & $4,07(3,50)$ & $0,00(0,00)$ & $0,89(2,02)$ & $5,03(3,88)$ \\
\hline Estudiante & $3(1,4)$ & $2(66,7)$ & $2,67(3,05)$ & $0,00(0,00)$ & $2,00(3,46)$ & $4,66(6,42)$ \\
\hline Hogar & $98(46)$ & $72(73,5)$ & $3,95(3,93)$ & $0,00(0,00)$ & $0,56(1.26)$ & $4,5(4,17)$ \\
\hline $\mathrm{F}(\mathrm{p})$ & & & $0,68(0.60)$ & $0,79(0,53)$ & $0,91(0,45)$ & $0,57(0.68)$ \\
\hline \multicolumn{7}{|c|}{ Ocupación del padre } \\
\hline Trabajador calificado & $23(10,8)$ & $12(52,2)$ & $2,65(3.67)$ & $0,00(0,00)$ & $0,61(1,61)$ & $3,39(4,52)$ \\
\hline Trabajador no calificado & $86(40,4)$ & $63(73,3)$ & $3,78(3,64)$ & $0,05(0,43)$ & $0,62(1,74)$ & $4,45(4,14)$ \\
\hline Comerciante & $21(9,9)$ & $17(81,0)$ & $4,38(3.41)$ & $0,00(0,00)$ & $0,52(1,28)$ & $4,95(3,48)$ \\
\hline Empleo libre & $40(18,8)$ & $31(77,5)$ & $4,80(4,30)$ & $0,00(0,00)$ & $0,68(1,16)$ & $5,47(4,48)$ \\
\hline Empleado por contrato & $5(2,3)$ & $1(20,0)$ & $0,60(1,34)$ & $0,00(0,00)$ & $0,20(0,44)$ & $0,80(1,30)$ \\
\hline Otro & $2(0,9)$ & $1(50,0)$ & $3,50(4,95)$ & $0,00(0,00)$ & $0,00(0,00)$ & $3,50(494)$ \\
\hline No aplica (no tiene padre) & $36(16,9)$ & $22(61,1)$ & $2,58(3,26)$ & $0,00(0,00)$ & $0,97(1,68)$ & $3,61(3.65)$ \\
\hline $\mathrm{F}(\mathrm{p})$ & & & $2,16(0,04)$ & $0,24(0,96)$ & $0,41(0,86)$ & $1,64(0,13)$ \\
\hline \multicolumn{7}{|c|}{ Acceso a los servicios } \\
\hline IMSS & $60(28,2)$ & $38(63,3)$ & $3,60(3,72)$ & $0,00(0,00)$ & $0,73(1,73)$ & $4,40(4,20)$ \\
\hline ISSTE & $15(7)$ & $9(60,0)$ & $2,53(3,29)$ & $0,27(1,03)$ & $0,93(2,54)$ & $3,73(5,11)$ \\
\hline PEMEX & $1(0,5)$ & $0(0,0)$ & $0,00(0,00)$ & $0,00(0,00)$ & $0,00(0,00)$ & $0,00(0,00)$ \\
\hline Otro & $41(19,2)$ & $30(73,2)$ & $3,80(4,11)$ & $0,00(0,00)$ & $0,63(1,33)$ & $4,46(4,15)$ \\
\hline No tiene & $95(44,6)$ & $70(73,7)$ & $3,82(3,67)$ & $0,00(0,00)$ & $0,60(1,32)$ & $4,44(3,93)$ \\
\hline$F(p)$ & & & $0,63(0,63)$ & $3,43(0,01)$ & $0,23(0,92)$ & $0,37(0,82)$ \\
\hline \multicolumn{7}{|c|}{ Vivienda } \\
\hline Propia & $81(38)$ & $60(74,1)$ & $3,79(3,66)$ & $0,05(0,44)$ & $0,83(1,84)$ & $4,69(4,34)$ \\
\hline Rentada & $78(36,6)$ & $55(70,5)$ & $3.72(3,87)$ & $0,00(0,00)$ & $0,46(1,11)$ & $4,19(3,99)$ \\
\hline Comparte & $52(24,4)$ & $32(61,5)$ & $3,38(3.37)$ & $0,00(0,00)$ & $0,73(1,61)$ & $4,19(4,00)$ \\
\hline Otra & $1(0,5)$ & $0(0)$ & $0,00(0,00)$ & $0,00(0,00)$ & $0,00(0,00)$ & $0,00(0,00)$ \\
\hline$F(p)$ & & & $0,44(0,71)$ & $0,53(0,65)$ & $0,83(0,47)$ & $0,61(0,60)$ \\
\hline
\end{tabular}


Tabla 3: Percepción que tienen las madres de la salud bucal de sus hijos su salud bucal según su estado civil y condiciones de vida.

\begin{tabular}{|c|c|c|c|c|c|c|c|c|c|c|}
\hline \multirow{3}{*}{$\begin{array}{l}\text { Variables de la madre } \\
\text { Estado Civil }\end{array}$} & \multicolumn{6}{|c|}{ Percepción que tienen las madres de la salud bucal de sus hijos } & & & \multirow{3}{*}{$\begin{array}{c}X^{2} \\
12,74 \\
\end{array}$} & \multirow{3}{*}{$\begin{array}{c}p \\
0,03\end{array}$} \\
\hline & \multicolumn{2}{|c|}{$\begin{array}{c}\text { Muy buena y buena } \\
100(46,95)\end{array}$} & \multicolumn{2}{|c|}{$\begin{array}{c}\text { Regular } \\
98(46,10) \\
\end{array}$} & \multicolumn{2}{|c|}{$\begin{array}{c}\text { Mala y muy mala } \\
15(7,04) \\
\end{array}$} & \multicolumn{2}{|c|}{ Total } & & \\
\hline & $\mathrm{N}$ & $(\%)$ & $\mathrm{N}$ & $(\%)$ & $\mathrm{N}$ & $(\%)$ & $\mathrm{N}$ & $\%$ & & \\
\hline Soltera & 12 & 38,71 & 17 & 54,84 & 2 & 6,45 & 31 & 14,55 & & \\
\hline Casada & 50 & 54,35 & 34 & 36,96 & 8 & 8,70 & 92 & 43,19 & & \\
\hline Divorciada & 2 & 66,67 & 1 & 33,33 & 0 & 0,00 & 3 & 1,41 & & \\
\hline Separada & 7 & 50,00 & 7 & 50,00 & 0 & 0,00 & 14 & 6,57 & & \\
\hline Unión Libre & 29 & 39,73 & 39 & 53,42 & 5 & 6,85 & 73 & 34,27 & & \\
\hline \multicolumn{11}{|c|}{ Condiciones de vida } \\
\hline \multicolumn{9}{|c|}{ Escolaridad de la madre } & 22,55 & 0,05 \\
\hline Primaria incompleta & 3 & 25,00 & 8 & 66,67 & 1 & 8,33 & 12 & 5,63 & & \\
\hline Primaria completa & 5 & 35,71 & 8 & 57,14 & 1 & 7,14 & 14 & 6,57 & & \\
\hline Secundaria & 27 & 37,50 & 39 & 54,17 & 6 & 8,33 & 72 & 33,80 & & \\
\hline Bachillerato & 22 & 55,00 & 14 & 35,00 & 4 & 10,00 & 40 & 18,78 & & \\
\hline Carrera técnica & 27 & 51,92 & 22 & 42,31 & 3 & 5,77 & 52 & 24,41 & & \\
\hline Licenciatura & 16 & 69,57 & 7 & 30,43 & 0 & 0,00 & 23 & 10,80 & & \\
\hline \multicolumn{9}{|c|}{ Escolaridad del padre } & 26,42 & 0,05 \\
\hline Primaria incompleta & 0 & 0,00 & 1 & 100,00 & 0 & 0,00 & 1 & 0,47 & & \\
\hline Primaria completa & 5 & 38,46 & 7 & 53,85 & 1 & 7,69 & 13 & 6,10 & & \\
\hline Secundaria & 23 & 43,40 & 27 & 50,94 & 3 & 5,66 & 53 & 24,88 & & \\
\hline Bachillerato & 37 & 48,68 & 32 & 42,11 & 7 & 9,21 & 76 & 35,68 & & \\
\hline Carrera técnica & 6 & 42,86 & 6 & 42,86 & 2 & 14,29 & 14 & 6,57 & & \\
\hline Licenciatura & 16 & 80,00 & 4 & 20,00 & 0 & 0,00 & 20 & 9,39 & & \\
\hline No aplica (no tiene padre) & 13 & 36,11 & 21 & 58,33 & 2 & 5,56 & 36 & 16,90 & & \\
\hline \multicolumn{9}{|c|}{ Ocupación de la madre } & 9,63 & 0,02 \\
\hline Empleada calificada & 17 & 50,00 & 17 & 50,00 & 0 & 0,00 & 34 & 15,96 & & \\
\hline Empleada no calificada & 27 & 52,94 & 22 & 43,14 & 2 & 3,92 & 51 & 23,94 & & \\
\hline Comerciante & 11 & 40,74 & 12 & 44,44 & 4 & 14,81 & 27 & 12,68 & & \\
\hline Estudiante & 1 & 33,33 & 2 & 66,67 & 0 & 0,00 & 3 & 1,41 & & \\
\hline Hogar & 44 & 44,90 & 45 & 45,92 & 9 & 9,18 & 98 & 46,01 & & \\
\hline \multicolumn{9}{|c|}{ Ocupación del padre } & 40,8 & 0,07 \\
\hline Trabajador calificado & 14 & 60,87 & 8 & 34,78 & 1 & 4,35 & 23 & 10,80 & & \\
\hline Trabajador no calificado & 44 & 51,16 & 39 & 45,35 & 3 & 3,49 & 86 & 40,38 & & \\
\hline Comerciante & 4 & 19,05 & 11 & 52,38 & 6 & 28,57 & 21 & 9,86 & & \\
\hline Empleo libre & 19 & 47,50 & 18 & 45,00 & 3 & 7,50 & 40 & 18,78 & & \\
\hline Empleado por contrato & 5 & 100,00 & 0 & 0,00 & 0 & 0,00 & 5 & 2,35 & & \\
\hline Otro & 1 & 50,00 & 1 & 50,00 & 0 & 0,00 & 2 & 0,94 & & \\
\hline No aplica (no tiene padre) & 13 & 36,11 & 21 & 58,33 & 2 & 5,56 & 36 & 16,90 & & \\
\hline \multicolumn{9}{|c|}{ Acceso a los servicios } & 19,98 & 0,03 \\
\hline IMSS & 30 & 50,00 & 27 & 45,00 & 3 & 5,00 & 60 & 28,17 & & \\
\hline ISSSTE & 11 & 73,33 & 3 & 20,00 & 1 & 6,67 & 15 & 7,04 & & \\
\hline PEMEX & 1 & 100,00 & 0 & 0,00 & 0 & 0,00 & 1 & 0,47 & & \\
\hline Otro & 15 & 36,59 & 21 & 51,22 & 5 & 12,20 & 41 & 19,25 & & \\
\hline No tiene & 42 & 44,21 & 47 & 49,47 & 6 & 6,32 & 95 & 44,60 & & \\
\hline
\end{tabular}


Tabla 3: Percepción que tienen las madres de la salud bucal de sus hijos su salud bucal según su estado civil y condiciones de vida. Cont.

\begin{tabular}{|c|c|c|c|c|c|c|c|c|c|c|}
\hline \multirow{2}{*}{ Variables de la madre } & \multicolumn{6}{|c|}{ Percepción que tienen las madres de la salud bucal de sus hijos } & \multirow{2}{*}{\multicolumn{2}{|c|}{ Total }} & \multirow[b]{2}{*}{$X^{2}$} & \multirow[b]{2}{*}{$\mathrm{p}$} \\
\hline & \multicolumn{2}{|c|}{$\begin{array}{c}\text { Muy buena y buena } \\
100(46,95)\end{array}$} & \multicolumn{2}{|c|}{$\begin{array}{c}\text { Regular } \\
98(46,10)\end{array}$} & \multicolumn{2}{|c|}{$\begin{array}{c}\text { Mala y muy mala } \\
15(7,04)\end{array}$} & & & & \\
\hline \multicolumn{9}{|c|}{ Vivienda } & 5,1 & 0,01 \\
\hline Propia & 38 & 46,91 & 38 & 46,91 & 5 & 6,17 & 81 & 38,03 & & \\
\hline Rentada & 36 & 46,15 & 34 & 43,59 & 8 & 10,26 & 78 & 36,62 & & \\
\hline Comparte & 24 & 46,15 & 26 & 50,00 & 2 & 3,85 & 52 & 24,41 & & \\
\hline Otra & 1 & 100,00 & 0 & 0,00 & 0 & 0,00 & 1 & 0,47 & & \\
\hline \multicolumn{9}{|c|}{ Sexo } & 2,34 & 0,01 \\
\hline Femenino & 51 & 50,50 & 44 & 43,56 & 6 & 5,94 & 101 & 47,42 & & \\
\hline Masculino & 49 & 43,75 & 54 & 48,21 & 9 & 8,04 & 112 & 52,58 & & \\
\hline \multicolumn{9}{|c|}{ Edad del preescolar } & 6,34 & 0,01 \\
\hline 4 años & 14 & 53,85 & 11 & 42,31 & 1 & 3,85 & 26 & 12,21 & & \\
\hline 5 años & 58 & 48,74 & 51 & 42,86 & 10 & 8,40 & 119 & 55,87 & & \\
\hline 6 años & 28 & 41,18 & 36 & 52,94 & 4 & 5,88 & 68 & 31,92 & & \\
\hline \multicolumn{9}{|c|}{ Última visita del niño al dentista } & 12,47 & 0,02 \\
\hline Menos de 3 meses & 20 & 47,62 & 20 & 47,62 & 2 & 4,76 & 42 & 19,72 & & \\
\hline De 3 a 6 Meses & 22 & 53,66 & 17 & 41,46 & 2 & 4,88 & 41 & 19,25 & & \\
\hline De 7 a 12 meses & 12 & 46,15 & 11 & 42,31 & 3 & 11,54 & 26 & 12,21 & & \\
\hline Mas de un año & 17 & 40,48 & 23 & 54,76 & 2 & 4,76 & 42 & 19,72 & & \\
\hline Nunca ha ido al dentista & 29 & 46,77 & 27 & 43,55 & 6 & 9,68 & 62 & 29,11 & & \\
\hline \multicolumn{9}{|c|}{ Motivo de la consulta } & 15,37 & 0,03 \\
\hline Alguna molestia al dolor & 15 & 34,09 & 23 & 52,27 & 6 & 13,64 & 44 & 20,66 & & \\
\hline $\begin{array}{l}\text { Continuar con algún trata- } \\
\text { miento }\end{array}$ & 10 & 45,45 & 11 & 50,00 & 1 & 4,55 & 22 & 10,33 & & \\
\hline Prevención & 36 & 53,73 & 30 & 44,78 & 1 & 1,49 & 67 & 31,46 & & \\
\hline Estética & 4 & 66,67 & 2 & 33,33 & 0 & 0,00 & 6 & 2,82 & & \\
\hline Otra & 6 & 50,00 & 5 & 41,67 & 1 & 8,33 & 12 & 5,63 & & \\
\hline Nunca ha ido al dentista & 29 & 46,77 & 27 & 43,55 & 6 & 9,68 & 62 & 29,11 & & \\
\hline \multicolumn{9}{|c|}{ Tipo se servicio odontológico utilizado } & 33,13 & 0,06 \\
\hline 0 & 1 & 50,00 & 1 & 50,00 & 0 & 0,00 & 2 & 0,94 & & \\
\hline IMSS & 5 & 41,67 & 7 & 58,33 & 0 & 0,00 & 12 & 5,63 & & \\
\hline ISSSTE & 4 & 66,67 & 2 & 33,33 & 0 & 0,00 & 6 & 2,82 & & \\
\hline$\overline{\text { SEDENA }}$ & 0 & 0,00 & 1 & 100,00 & 0 & 0,00 & 1 & 0,47 & & \\
\hline PEMEX & 1 & 100,00 & 0 & 0,00 & 0 & 0,00 & 1 & 0,47 & & \\
\hline Privado & 40 & 51,28 & 33 & 42,31 & 5 & 6,41 & 78 & 36,62 & & \\
\hline Secretaría de Salud & 11 & 40,74 & 14 & 51,85 & 2 & 7,41 & 27 & 12,68 & & \\
\hline Otro & 9 & 36,00 & 14 & 56,00 & 2 & 8,00 & 25 & 11,74 & & \\
\hline Nunca ha ido al dentista & 29 & 47,54 & 26 & 42,62 & 6 & 9,84 & 61 & 28,64 & & \\
\hline \multicolumn{9}{|c|}{ Veces de cepillado al día } & 33,71 & 0,05 \\
\hline 1 & 30 & 48,39 & 31 & 50,00 & 1 & 1,61 & 62 & 29,11 & & \\
\hline 2 & 43 & 45,74 & 41 & 43,62 & 10 & 10,64 & 94 & 44,13 & & \\
\hline 3 & 21 & 53,85 & 17 & 43,59 & 1 & 2,56 & 39 & 18,31 & & \\
\hline 0 & 6 & 33,33 & 9 & 50,00 & 3 & 16,67 & 18 & 8,45 & & \\
\hline \multicolumn{9}{|c|}{ Consumo de dulces } & 12,64 & 0,02 \\
\hline Todos los días & 14 & 36,84 & 19 & 50,00 & 5 & 13,16 & 38 & 17,84 & & \\
\hline La mayoría de los días & 42 & 47,73 & 40 & 45,45 & 6 & 6,82 & 88 & 41,31 & & \\
\hline Una vez por semana & 27 & 50,94 & 23 & 43,40 & 3 & 5,66 & 53 & 24,88 & & \\
\hline Rara vez & 16 & 48,48 & 16 & 48,48 & 1 & 3,03 & 33 & 15,49 & & \\
\hline Nunca & 1 & 100,00 & 0 & 0,00 & 0 & 0,00 & 1 & 0,47 & & \\
\hline
\end{tabular}


Tabla 4: Percepción de la salud bucal por parte de las madres de preescolares (PSB) y el índice ceo-d de sus hijos.

\begin{tabular}{|c|c|c|c|c|c|c|}
\hline Entrevista & Edad (años) & Escolaridad & Ocupación & PSB & ceo-d & No. de hijos \\
\hline \multicolumn{7}{|l|}{ BUENA } \\
\hline E5 & 27 & $\begin{array}{l}\text { Carrera } \\
\text { Técnica }\end{array}$ & $\begin{array}{c}\text { Hogar- } \\
\text { autoempleo }\end{array}$ & Buena & 0,25 & 2 \\
\hline E6 & 27 & $\begin{array}{l}\text { Primaria } \\
\text { incompleta }\end{array}$ & Hogar & Buena & 0,70 & 5 \\
\hline E1 & 32 & $\begin{array}{c}\text { Carrera } \\
\text { Profesional }\end{array}$ & Hogar & Buena & 0,00 & 2 \\
\hline E7 & 39 & $\begin{array}{l}\text { Carrera } \\
\text { Técnica }\end{array}$ & $\begin{array}{c}\text { Hogar } \\
\text { autoempleo }\end{array}$ & Buena & 0,10 & 3 \\
\hline \multicolumn{7}{|l|}{ REGULAR } \\
\hline E9 & 23 & Secundaria & Hogar & Regular & 0,50 & 2 \\
\hline E8 & 42 & Secundaria & Hogar & Regular & 0,00 & 1 \\
\hline E2 & 32 & Secundaria & Hogar & Regular & 0,15 & 4 \\
\hline E3 & 32 & Secundaria & Hogar & Regular & 0,50 & 2 \\
\hline \multicolumn{7}{|l|}{ MALA } \\
\hline E4 & 33 & Secundaria & Empleada & Mala & 0,55 & 2 \\
\hline E10 & 48 & Carrera técnica & $\begin{array}{c}\text { Empleada y } \\
\text { hogar }\end{array}$ & Mala & 0,54 & 4 \\
\hline E11 & 23 & Secundaria & Hogar & Mala & 0,60 & 1 \\
\hline E12 & 36 & Preparatoria & Comerciante & Mala & 0,70 & 2 \\
\hline
\end{tabular}

enfermedad más reconocida es la caries dental la cual es detectada mediante la identificación visual, olfativa y sensitiva/ táctil. El dolor en sí mismo fue interpretado como enfermedad; el dolor de muelas, ver "dientes picados" significa para las madres que existe una enfermedad bucal. Las restauraciones y amalgamas fueron señaladas como signos de enfermedad.

Reconocimiento y percepción de la enfermedad.

Las respuestas culturales más frecuentes fueron: huelen mal, se ven hoyos, puntos negros, manchas blancas, se le ven huecos, manchas cafés, hoyos rojos, puntos negritos y por el dolor. Las afecciones bucodentales son percibidas principalmente cuando causan dolor, es decir, cuando la enfermedad se encuentra en una etapa avanzada.

Percepción del valor social, cultural y biológico atribuido a la boca.

Para las madres entrevistadas la boca tiene un valor biológico y social alto, el atributo más destacado es la apariencia. Una de las funciones biológicas descrita fue la de comer, alimentarse, así como otras socialbiológicas tales como sentir la comida, los sabores, hablar, comunicarse con otros, para no verse mal, para expresarse, para sentirse seguras, para poder sonreír. 
El valor estético atribuido a la boca es percibida por las madres como elemento central de la información difundida a través de los medios de comunicación masiva; tener una "sonrisa estética" es signo de buena salud bucal, haber perdido algún diente genera rechazo y significa una mala salud bucal.

\section{Estrategias de auto atención bucodentales.}

Los conocimientos que poseen las madres para atender enfermedades bucodentales han sido obtenidos a través de la experiencia y de las creencias familiares. Se identifican conocimientos de auto atención que son practicados por las madres antes de considerar visitar al dentista, entre ellos destacan el cepillado dental, el uso del hilo y enjuague bucal, la automedicación y el uso de remedios caseros como el clavo, infusiones, bicarbonato, corteza de encino, sal de grano, algodón con tequila, tortilla quemada, jabón y canela. Para las madres una enfermedad bucal o una lesión por caries que genere dolor se traduce en la necesidad de atención odontológica y esto a su vez se asocia al gasto de dinero. Del grupo de cuatro madres que calificaron la salud bucal de sus hijos como buena, únicamente en un caso el resultado de la revisión bucal confirmó dicha percepción. La automedicación es una respuesta que las madres dan a las enfermedades bucodentales, principalmente cuando se trata de aminorar una situación de dolor. La elección de los medicamentos a emplear la realizan de acuerdo con anuncios televisivos. Los medicamentos más mencionados fueron el ibuprofeno, diclofenaco, paracetamol y aspirina.

Relación con los servicios odontológicos

La mayoría de las madres están afiliadas al seguro popular o al servicio de gratuidad que ofrece el gobierno de la Ciudad de México. Reconocen que los tratamientos ofrecidos por el seguro popular se limitan a la prevención, profilaxis, a curaciones menores y a la promoción de información general sobre las enfermedades bucodentales. En general la percepción de los servicios odontológicos fue negativa en relación con su costo. La significación más importante fue económica pues representa un gasto, un lujo, algo que sale de sus posibilidades. Relacionan la calidad de la atención con su costo, si los servicios odontológicos son de costo moderado a bajo piensan que son de baja calidad.

Relación de las enfermedades bucodentales y sus condiciones de vida.

La vivienda, el trabajo y la alimentación fueron las principales determinantes que las madres asociaron con sus condiciones de vida y su estado de salud bucodental. La vivienda fue asociada con insalubridad, por ejemplo, si tenía piso de tierra. El trabajo representa para ellas menos atención para sus hijos, pero mayor ingreso económico. Atribuyen las causas de sus enfermedades bucodentales a malos hábitos y a comportamientos individuales. La alimentación se asocia con las enfermedades bucales pues reconocen el daño potencial de alimentos procesados y con altas concentraciones de sal y azúcar. Al preguntar cuáles son las necesidades que no pueden cubrir, mencionaron la ropa, los zapatos, la despensa, la vivienda, la atención médica y odontológica.

\section{Discusión}

La caries dental en los niños de edad preescolar tiene una prevalencia 
elevada, este hecho se reflejará en su vida adulta limitando el desarrollo de sus capacidades. La caries dental es una enfermedad compleja resultado de múltiples determinaciones que incluyen aspectos biológicos y socioculturales, para su estudio es necesario reconocer la importancia de elementos como la percepción de la enfermedad, las condiciones de vida, entre otros, que se discuten a continuación.

Condiciones de vida de las madres de los preescolares.

Las madres de preescolares del jardín de niños Olmedo se encuentran en un rango de edad de entre 20 y 51 años. Son vulnerables a las enfermedades bucales debido a sus condiciones de vida, que en este estudio evidenciaron bajos ingresos económicos, limitado acceso a los servicios de salud y bajo nivel educativo. ${ }^{16}$ El $46 \%$ de las madres se ocupa de su hogar, 42,6\% trabaja de manera formal o irregular (Tabla 2bis). Estudios realizados en México demuestran que existe una correlación entre la prevalencia de caries dental y el desempleo. ${ }^{17}$ Los preescolares de las madres que se dedican al hogar presentaron mayor prevalencia de caries en comparación con los hijos de madres que trabajan como profesionistas. ${ }^{10}$

No se encontró asociación entre nivel de escolaridad de las madres y la prevalencia de caries dental en sus hijos. En el caso de la escolaridad de los padres, otros estudios han encontrado que la escolaridad formal baja, se relaciona con actitudes negativas respecto a la higiene de sus hijos. En el presente estudio las madres de los preescolares entrevistadas no continuaron con la educación formal debido a limitaciones socioeconómicas; la relación entre limitantes socioeconómicas con la prevalencia e incidencia de caries dental observada en este trabajo se ha encontrado previamente por Navas et al ${ }^{18}$ así como por Medina. ${ }^{19}$

Al no contar con empleos formales, el $44,6 \%$ de las madres no tiene derecho a algún servicio de seguridad social (Tabla 2bis). La atención a la que tienen acceso la mayoría de los preescolares y sus padres corresponde a la Secretaría de Salud que ofrecía atención a población abierta a través del Seguro Popular (en el año 2020, este servicio fue sustituido por otro esquema de atención, denominado Instituto de Salud para el Bienestar). Para la atención de la población de la zona existe una Clínica de Medicina Familiar del Instituto de Seguridad Social y Servicios Sociales de los Trabajadores del Estado (ISSSTE), con una población de sujetos de atención potencial de 150.584 personas. ${ }^{20}$ Los resultados muestran que la existencia de estos servicios públicos de salud no garantiza que sean usados o que se tenga acceso a ellos (Tabla 1bis).

\section{Servicios odontológicos.}

E1 29,1\% de los preescolares no ha visitado nunca a un dentista (Tabla 1bis), esto puede estar relacionado con la edad del preescolar y con la poca importancia que se le da a la dentición primaria. En relación con las necesidades de salud bucal de los niños, la utilización de servicios odontológicos de salud se encontró asociada a la economía familiar, la educación de los padres, el orden de los niños al nacer, así como su sexo. El 31,5\% de las madres dijo acudir a una consulta odontológica por prevención (Tabla 1bis), lo cual debería reflejarse en evaluaciones bucodentales categorizadas como libres de caries, sin embargo, en dichos casos la evaluación bucal de los preescolares se clasificó como mala o con presencia de 
caries. El 20,7\% indicó que asistió a una consulta motivado por una molestia o dolor, dicha causa es altamente frecuente en niños de cinco años. ${ }^{21}$ Los resultados sugieren que los programas preventivos de las instituciones públicas y la difusión de prácticas como el consumo de pastas, hilos y cepillos dentales son insuficientes por sí mismos para la disminución de la prevalencia de caries dental.

Prácticas de higiene bucal de los preescolares.

El $44,1 \%$ de las madres refieren, que los niños cepillan sus dientes dos veces al día (Tabla 1 bis), sin embargo, esto no se refleja en la salud bucodental de sus hijos. La falta higiene bucal es una de las principales causas a las que se atribuye la caries dental, si es deficiente existen más probabilidades de desarrollar caries y otras patologías bucodentales. ${ }^{17}$ Un resultado importante es la frecuencia con la que los preescolares consumen dulces, pues es otra de las causas importantes para el desarrollo de caries dental (Tabla 3bis). El $41 \%$ refiere que su consumo es la mayoría de los días, y un 17,8\% refiere que todos los días, la mitad de la población encuestada refiere que sus hijos consumen dulces con mucha frecuencia. Este resultado sugiere que el consumo de estos productos no está relacionado por las madres como un factor que promueve el desarrollo de caries dental; ${ }^{22,}{ }^{23}$ sólo relacionan al cepillado, al uso de pasta e hilo dental, como hábitos importantes para tener una buena salud bucal. Aspectos como la alimentación y el consumo excesivo de carbohidratos no son percibidos como elementos determinantes para el desarrollo de la caries dental. Las madres de los preescolares creen que los dulces, el pan, los cereales "de caja", los jugos y refrescos son parte de una alimentación normal (que no produce daño a la boca), no perciben sus efectos en la salud en general; ignoran el contenido de azucares y su relación con diversas afectaciones a la salud bucal de sus hijos.

\section{Índice coe-d.}

De acuerdo con la OMS la población de preescolares estudiada se encontraría en un nivel moderado de caries dental $(4,34)$. A pesar de que el índice ceo-d no permite la distinción entre los dientes indicados para extracción o perdidos por caries, posibilita la identificación de problemas dentales pasados, presentes y futuros, lo cual puede favorecer la creación de planes de atención bucal. Las limitaciones del índice son que, al ser un promedio, se suaviza el resultado perdiendo de vista lo alarmante que puede ser la presencia de caries en una población. ${ }^{8,24}$ Los patrones de atención regular están positivamente asociados con el número de dientes obturados (componente od o OD), y negativamente asociado con el número de dientes perdidos (componente ed o PD). Así, individuos con el mismo valor de ceo-d o CPOD pueden tener diferente patrón de experiencia de caries, ${ }^{24}$ sin que dichas experiencias se consideren en la interpretación de los resultados. Otra consideración es que el índice valora de la misma forma una lesión por caries (componente cd o CD), con diversos grados de destrucción dental, que requerirán de restauraciones diferentes. De igual forma trata una obturación simple o compuesta (componente diente obturado), sin considerar la severidad del daño causado por un mismo componente. ${ }^{8}$ A pesar que de acuerdo con el índice ceo-d, el nivel de caries en los preescolares evaluados en el presente estudio puede clasificarse como moderado, es necesario considerar que la enfermedad está presente en edades tempranas, que se observa la pérdida de dientes, que la atención especializada es baja o nula y que es muy probable que los preescolares continúen desarrollando la enfermedad a lo largo de su vida. 
Prevalencia de caries dental.

Las madres refieren que sus hijos(as) se cepillan los dientes por lo menos dos veces al día (Tabla 3bis). Sin embargo, al contrastar estos resultados con la prevalencia de la enfermedad, 69\% de los preescolares presentan caries dental y sólo el $31 \%$ no presentan la enfermedad, se observa que este resultado no concuerda con la percepción y lo referido por las madres en cuanto al cepillado de sus hijos. Si los preescolares se cepillaran dos veces al día, dicha práctica debería reflejarse en una menor prevalencia de placa y de caries dental. La aparición de caries dental no depende únicamente de las prácticas de cepillado sino de una serie de determinantes más complejas. ${ }^{25}$

Cabe mencionar que una de las políticas públicas en salud bucal en México para la prevención de caries dental es la fluorización de la sal de mesa, las pastas dentales comerciales y de fácil acceso a la población contienen fluoruro, sin embargo, la prevalencia de caries dental en la población infantil mexicana es alta.

Para modificar la prevalencia de la caries y su oportuna atención, es necesario ampliar el marco de acción del profesional de la salud y de las políticas y los programas de salud bucal, reconociendo la importancia de los aspectos sociales y culturales que actúan en la determinación de la enfermedad.

\section{Conclusiones}

En el presente trabajo se estudió la prevalencia de caries dental en niñas y niños en edad preescolar en relación con la percepción de sus madres respecto a la enfermedad y la salud bucal. Los resultados mostraron que la prevalencia de caries dental observada en los niños en edad preescolar fue de $69 \%$ con un índice ceo-d fue de 4,34 y que más de la mitad de las madres, 53,4\%, calificaron la salud bucal de sus hijos como "regular", concordando con los parámetros establecidos por la OMS, que clasifican a la caries dental como "moderada" cuando los valores de dientes afectados se sitúan entre 2,7 y 4,4 dientes afectados en promedio, aunque es de notar que, en este caso, el promedio se ubicó en el límite superior.

El estudio cualitativo permitió comprender cómo las madres entrevistadas perciben la enfermedad a través de tres sentidos: mediante la identificación visual (color del diente), olfativa (olor bucal) y sensitiva/ táctil (dolor, generalmente severo). En el caso del dolor, éste representa la manifestación más crítica de las lesiones dentarias por caries avanzada y esto permite suponer que, si bien las madres pueden identificar la enfermedad, no logran hacerlo tempranamente, ni reconocen su grado de severidad. Las madres entrevistadas, consideraron a la caries dental como una enfermedad con poca repercusión en la salud general y el desarrollo de sus hijos; esta percepción impide el uso de servicios odontológicos preventivos de manera oportuna. Así mismo acudir al odontólogo implica un costo que no corresponde con la capacidad de pago dado el bajo ingreso familiar del grupo estudiado y por lo tanto lo hacen cuando no queda otra alternativa para aliviar el dolor. La actitud hacia estos servicios fue ambivalente, por un lado negativa en relación con su costo y al limitado acceso a los servicios odontológicos públicos, pero por el otro, predominó la idea de que al atenderse la caries se "cura", lo cual no corresponde con alguna evidencia científica. 
Las prácticas de autocuidado, basadas en el uso de remedios caseros y la automedicación para aliviar el dolor, contribuyen a postergar el uso de los servicios de salud bucal aumentando con ello el riesgo a futuro de mayor número de dientes afectados, necesidades no cubiertas y manifestaciones adversas como dolor y otras afecciones en la dentadura temporal y permanente.

Adicionalmente, la percepción de las madres sobre la salud bucal de sus hijos se ve influida por sus representaciones sociales, en donde la noción estética, aprendida a través de la información generada por medios de comunicación masiva que promueven el consumo de pastas, cepillos y enjuagues bucales, que si bien contribuyen a incentivar las prácticas de cepillado dental, enfatizan las "sonrisas saludables"; lo que significa que, mientras no haya visibles huellas de deterioro dental, no se presta suficiente atención a los problemas bucales de los niños. Esta situación se agrava con la percepción de que el consumo de dulces, refrescos y otras golosinas como mecanismo de recompensa, repercute en la salud bucal de las niñas y los niños. Es notable en México que estos alimentos azucarados altamente cariogénicos tienen una promoción y venta masiva, e incluyen mensajes atractivos y dirigidos para los niños y niñas, pero que también parecieran alcanzar su objetivo comercial en a las madres.

Se puede concluir que la salud bucal de este grupo de niñas y niños en edad prescolar estudiado estuvo relacionada con las condiciones socioeconómicas, el nivel de escolaridad, la insuficiente disponibilidad de servicios odontológicos accesibles, así como con las ideas, representaciones y respuestas culturales de las madres influidas fuertemente por mensajes publicitarios que, en general, envían información contradictoria y muchas veces nociva para la salud. Lo que no se encontró y destaca por ello, es la presencia de conceptos derivados de mensajes claros de promoción y educación para la salud basados en el conocimiento científico actual de la odontología, ya sea a través de programas de salud institucionales, de asociaciones profesionales o de los propios medios de comunicación que contribuyeran a desarrollar capacidades de autocuidado efectivas hacia a los niños y niñas, las madres y padres y distintos grupos de edad.

Requieren ser considerados otras determinantes sociales, también debe fortalecerse la sensibilización en la profesión odontológica para generar una práctica con mayor pertinencia social y por tanto con mayor impacto frente a los problemas de salud bucal de la población infantil.

\section{Agradecimientos}

Al CONACYT por la beca (37865) otorgada a la Mtra. Natalia Odeth Santos Madrigal. Al Dr. Iván Pável Moreno-Espíndola por el apoyo en la elaboración del presente artículo.

\section{Referencias bibliográficas}

1. Langdon EJ, Wiik FB. Antropología, salud y enfermedad: una introducción al concepto de cultura aplicado a las ciencias de la salud. Rev Latino-Am Enfermagem. 2010; 18(3):177-185.

2. Sacchi M, Hausberger M, Pereyra A, Percepción del proceso salud-enfermedad-atención y aspectos que influyen en la baja utilización del sistema de salud, en familias pobres de la ciudad de Salta. Rev Salud Colectiva. 2007; 3(3):271-283. 
3. Cubero SA. Lorido CI, González H, Ferrer GM, Zapata CM, Ambel S.J. Prevalencia de caries dental en escolares de educación infantil de una zona de salud con nivel socioeconómico bajo. Rev Pediatr. Aten Primaria. 2019 Jun 21(82):e47-e59.

4. Olaiz FG, Rivera DJ, Shamah LT, Rojas R, Villalpando HS, Hernández AM, Sepúlveda AJ. Encuesta Nacional de Salud y Nutrición 2006. Instituto Nacional de Salud Pública. 2006.

5. Bayona EE. Enfermedad y pobreza en la Sierra de Chiapas. Liminar. 2011; 9(2):93-115.

6. Lara FN, Delgadillo GH, Morales ES, Garduño AMA, Pulido NM. Necesidades insatisfechas de atención odontológica en trabajadores de la costura en México D.F. Rev Salud de los Trabajadores. 2011; 19(1):35-46.

7. Tinanoff N, Palmer CA. Dietary determinants of dental caries and dietary recommendations for preschool children. J Public Health Dent. 2000; 60(3) 197-206.

8. Segovia VA., Estrella RR, Medina SCE, Maupomé G. Severidad de caries y factores asociados en preescolares de 3-6 años de edad en Campeche, México. Rev Salud Pública. 2005; 7(1): 56-69.

9. Molina FN, Durán MD, Castañeda CE, Juárez LMLA. La caries y su relación con la higiene oral en preescolares mexicanos. Gac Méd Méx. 2015; 151(4):485-490.

10. Adriano AMP, Caudillo JT. Caries dental en escolares, su relación con variables socioprofesionales de los padres, México. Rev Costarric Salud Pública. 2008; 17 (33):24-31.

11. Lagarde RM. Los cautiverios de las mujeres: madresposas, monjas, putas, presas y locas. UNAM: Col. Posgrado. 2005.

12. González OC. Apuntes acerca de la representación. Instituto de Investigaciones Fiolológicas, UNAM. México; 2005.

13. Organización Mundial de la Salud. Oral Health Surveys, Basic methods. Fourth Ed. World Health Organization, Geneva; 1997. OMS Encuestas de Salud Bucodental. Métodos Básicos Cuarta Ed. 1997 Ginebra, Suiza pp 21-34

14. Alvarez GJ. Cómo hacer investigación cualitativa: Fundamentos y Metodología. Paidós. 2003; 2 (003)

15. Creswell J. Qualitative inquiry and research design. Choosing among five traditions. Londres. 1988.

16. Bronfman M, Tuirán R. La desigualdad social ante la muerte: clases sociales y mortalidad en la niñez. En Memorias del Congreso Latinoamericano de Población y Desarrollo. 1984. p. 53-57.

17. Irigoyen M, Zepeda M, Sánchez L, Molina N. Prevalencia e incidencia de caries dental y hábitos de higiene bucal en un grupo de escolares del sur de la Ciudad de México: Estudio de seguimiento longitudinal. Rev ADM. 2001; 58(3):98-104.

18. Navas PR, Rojas MT, Zambrano O, Álvarez CJ, Santana Y, Viera N. Salud bucal en preescolares: su relación con las actitudes y nivel educativo de los padres. Interciencia. 2001; 27(11):631-634.

19. Medina CE, Maupomé G, Pelcastre-Villafuerte B, Avila-Burgos L, Vallejos-Sánchez AA, Casanova-Rosado AJ. Desigualdades socioeconómicas en salud bucal: caries dental en niños de seis a 12 años de edad. Rev invest clín. 2006; 58(4):296-304.

20. ISSSTE. Catálogo único de unidades médicas. (2016 Octubre 1). Disponible en: URL:http://www.gob.mx/ cms/uploads/attachment/file/88139/Catalogo_Unico_de_Unidades_Medicas.pdf

21. Bravo SB. La caries dental como precursor de urgencia estomatológica en niños de círculo infantil. Tesis de Maestría Instituto Superior de Ciencias Médicas de la Habana. 2007.

22. Jensen ME. Diet and dental caries. Dent Clin North Am. 1999; 46(4):615-33.

23. Scottish Intercollegiate Guidelines Network. Preventing Dental Caries in Children at High Caries Risk: Targeted prevention of dental caries in the permanent teeth of 6-16 year olds presenting for dental care. SIGN Executive Royal College of Physicians 9 Queen Street Edinburgh. 2000.

24. Sheiham A, Maizels J, Maizels A. New composite indicators of dental health. Community Dent Health. 1987; 4:407-414.

25. Abadía BCE. Pobreza y desigualdades sociales: un debate obligatorio en salud oral. Acta Bioethica. 2006; 12(1):12.

26. Giménez G. La concepción simbólica de la cultura. En Teoría y análisis de la cultura. México. Conaculta; 2005. p. 67-87.

27. Petersen PE. The World Oral Heal th Report 2003. Geneva: WHO; 2003.

Recibido: : 23/10/2019

Aceptado: 07/05/2020

Correspondencia: Natalia O. Santos, correo: nataliaodeth@gmail.com 